\title{
EXPLOTACIÓN FAUNÍSTICA E INCORPORACIÓN DE GANADO DOMÉSTICO EUROASIÁTICO: EL REGISTRO ARQUEOLÓGICO EN LA FRONTERA NORDPATAGÓNICA.
}

\author{
Adolfo Gil*; Gustavo Neme**; Víctor Durán*** \\ *CONICET-Departamento de Antropología; Museo de Historia Natural de San Rafael. Parque Mariano Moreno \\ (5600) San Rafael, Mza.afgil1@infovia.com.ar; \\ ${ }^{*}$ CONICET-Departamento de Antropología; Museo de Historia Natural de San Rafael. Parque Mariano Moreno \\ (5600) San Rafael, Mza. ananeme@infovia.com.ar; \\ ${ }^{* * * C O N I C E T-F a c u l t a d}$ de Filosofía y Letras. Universidad Nacional de Cuyo. (Mendoza). CC: 345 (5500). \\ Mendoza, Argentina.duranvic@logos.uncu.edu.ar
}

\section{Resumen}

Se aborda el tema de la incorporación de ganado doméstico euroasiático en contex to de cazadores-recolectores del sur de Mendoza (nordpatagonia). Para ello se presenta información etnohistórica y arqueológica. Se enfatiza en la información arqueofaunística evaluando la proporción de especies domésticas respecto a las silvestres y las tendencias de la fauna silvestre en el lapso de los últimos 500 años. La información presentada es concordante con la hipótesis que propone que la fauna doméstica fue cuantitativamente relevante sólo a partir del siglo XIX y que la incorporación de esta produjo un impacto en el ecosistema reflejado en un aumento en la proporción de fauna silvestre pequeña respecto a la fauna silvestre mediana y grande.

Palabras Claves: Arqueofauna - Hispano indígena - Mendoza - Subsistencia.

\begin{abstract}
This paper discuses the euroasiatic domestic animal incorporation in the hunter-gatherers context of Southern Mendoza (North Patagonia). In order to discuss this topic etnohistorical and archaeological information is presented. The archaeofaunistic data shows the proportion of domestic vs. wild species and the wild faunal trends along the last 500 years. On one hand, the data shows that the domestic fauna was only quantitatively significant since XIX century. On the other hand, the incorporation of this fauna produced impact in the ecosystem showing increased in the proportion of small size wild fauna in respect to medium and large wild fauna.
\end{abstract}

Key word: Archaeofauna-Mendoza-Subsistence

\section{INTRODUCCIÓN}

La incorporación de ganado doméstico es un tema de importancia tanto para entender los cambios socioculturales como los del ambiente natural de una región (Yellen 1990).

Este trabajo aborda el tema focalizado en el sur mendocino, en donde tal incorporación se realizó con ganado euroasiático a partir del siglo XVII (Cabrera 1929). Recientemente algunos trabajos proponen hipótesis sobre la cronología, el modo y el impacto que ocasionó tal incorporación en la región (Prieto y Abraham 1993-1994; Durán 2000). Estas hipótesis han sido discutidas principalmente con documentación escrita (Actas Capitulares, datos de crónicas, informes de expediciones militares, primeros de relevamiento de población y recurso, entre los más significativos). Pero estos documentos no tienen la misma importancia a lo largo del período involucrado (siglo XVI-siglo XIX), no han cubierto los procesos de los diferentes sectores del territorio, y presentan diferente grado de fidelidad y resolución res- 
pecto a la información pertinente a la incorporación de ganado euroasiático. Por ello, utilizando una vía de análisis alternativa, este artículo focaliza la discusión principalmente basada en el registro arqueológico, específicamente arqueofaunístico, que presenta una escala de resolución y procesos de formación distintos al registro histórico (Schiffer 1987). Con este registro se intentará corroborar algunas de las hipótesis planteadas y generar nuevas expectativas vinculadas a los cambios económicos y ambientales ocurridos posterior al contacto con poblaciones europeas.

\section{EL PERIODO HISPANO INDIGENA Y LA EXPLOTACION DEL GANADO: EL REGISTRO ETNOHISTORICO}

El registro histórico de los siglos XVI a XIX muestra que el sur de la provincia de Mendoza fue ocupada por puelches y pehuenches y que los mismos experimentaron profundos cambios a partir del contacto hispano-indígena (Durán 2000; Prieto y Abraham 1993-1994). Los puelches ocuparon en principio la planicie oriental y el piedemonte oriental de la cordillera y los pehuenches los valles interandinos. A partir del siglo XVIII, estos últimos también se hacen con el control de una buena parte de la planicie oriental (Durán 1996). En otros trabajos, se ha dividido a estos tiempos de contacto entre indígenas y españoles en tres períodos: 1-cazadores-recolectores del siglo XVI, 2-cazadores-ecuestres del siglo XVII y 3pastores y mercaderes de los siglos XVIII y XIX (Durán 1999, 2000).

Las evidencias del paso de una organización social de bandas a otra de tipo tribal, o de una economía cazadora-recolectora a otra mixta en la que se incluye el pastoreo y el tráfico de bienes diversos, se observan claramente en la documentación histórica (Michieli 1978; Prieto 1984; Durán 1994). También se encuentra en ella, para finales del período (siglos XVIII y XIX) pruebas del funcionamiento de grandes redes de intercambio (Mandrini 1991), que vinculaban a los distintos grupos indígenas entre sí, con la sociedad hispano-criolla y con un sistema que había adquirido una dimensión mundial. La participación tanto de puelches como de pehuenches en estas redes es clara en la documentación histórica (Durán 1996, 1999) y también los datos que indican que habían incorporado en sus dietas alimentos de origen externo (yerba, azúcar, trigo, vacas, caballos, cabras, ovejas, entre otros). Estos bienes se introducían o, cuando era factible, los producía la misma sociedad.

La integración a un mercado macro-regional provocó cambios de importancia en la cultura de puelches y pehuenches. La documentación de fines del siglo XVIII (Durán 1996, 2000) describe a estos últimos criando ganado bovino, equino, ovino y caprino, y dirigiendo el tráfico de bienes desde y hacia Chile. Indudablemente ya no se trataba de bandas de cazadores-recolectores, sino de poblaciones con una mayor integración política, una incipiente jerarquización social y una economía basada ya no sólo en la caza y recolección, sino también en el comercio y el pastoreo (Durán 1996, 2000). La actividad pecuaria incluía la cría de ganado mayor (vacuno y equino) y menor (ovejas y cabras). Los pehuenches y otras parcialidades que ocuparon el sur y centro de Mendoza fueron excelentes criadores de ovejas. Su producción de lana era aprovechada dentro de cada grupo para elaborar textilería, sobre todo ponchos y mantas. Para el período indicado, los ponchos y mantas pehuenches se habrían transformado en bienes muy codiciados. Sólo la sociedad hispano-criolla recibía miles por año (León Solís 1991; Villalobos 1989; Durán 1996; Prieto et al. 1999) y en contrapartida enviaba una cantidad considerable de textilería y chucherías de origen europeo a los asentamientos indígenas. 
También la documentación del siglo XIX muestra claramente que, más allá de la frontera, la cría y el manejo de ganado fueron actividades económicas importantes. Al respecto cabe destacar las cifras que aporta el Censo Nacional de 1864, el cual consigna 30.000 vacunos, 20.000 equinos, 60.000 ovejas y 1.000 cabras como pertenecientes a los indígenas (Prieto y Abraham 1993-1994:23). Según Prieto y Abraham (1993-1994: Figura 6) las zonas con mayor actividad ganadera indígena durante ese período fueron el valle del río Atuel en su curso medio y bajo, los faldeos orientales del Cerro Nevado y el valle del río Grande.

\section{EL PERIODO HISPANO INDIGENA Y LA EXPLOTACION FAUNISTICA: EL REGISTRO ARQUEOLOGICO}

En el presente estudio se considera el registro arqueológico depositado desde el siglo XVI y/o fechados en los últimos 500 14C años AP. El registro de este rango temporal permite explorar la importancia y características de la incorporación del ganado euroasiático doméstico entre las poblaciones del sur mendocino. En ese sentido, y según las cronologías absolutas y relativas para el sur de Mendoza, se presenta la información proveniente de los sitios Los Leones-6, Agua de Los Caballos-1, Los Peuquenes, Ojo de Agua, Alero Puesto Carrasco y Cueva de Luna (Figura 1). El tema es abordado mediante la consideración de la presencia y abundancia taxonómica registrada arqueológicamente, que en algún grado reflejaría la explotación faunística realizadas por las poblaciones recientes. Para el análisis faunístico se utilizó la metodología previamente aplicada en conjuntos arqueofaunísticos de la región (Neme et al. 1997; Neme et al. 1998; Neme y Gil 2002) con los criterios allí explicitados. A pesar de ello es necesario algunas aclaraciones. Las determinaciones se basaron en análisis comparativos con las colecciones de referencias del Museo de Historia Natural de San Rafael, con las excepciones detalladas en los trabajos citados (Neme et al. 1996; Neme et al. 1999; Neme y Gil 2002). Debido al alto índice de fragmentación se ha considerado al NISP (Number Identified Specimen) como la unidad de cuantificación más confiable, aunque un caso especial lo constituyen los dasipodidos. En éstos no se incluyeron las placas dérmicas en la cuantificación del NISP, aunque si en la tabla de identificación taxonómica, ya que dificultan las comparaciones intra e interconjunto por su sobrerepresentación. En éstos casos el NISP se basa solo en los especimenes del esqueleto interno aunque en casos donde solo se registran placas se coloca un NISP de 1 con el fin de evitar sobreestimaciones que dificulten la comparación entre los conjuntos analizados.

\section{Sitio Los Leones 6 (Lole-6).}

Los Leones-6 (Lole-6) es un sitio a cielo abierto que se emplaza en el Area El Nevado, sector SE de Mendoza. Está localizado en la margen izquierda del zanjón seco Casa de

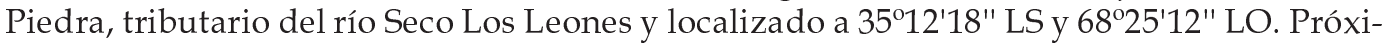
mo a Lole-6 se ubicaron varios reparos rocosos, alguno de ellos han sido excavados (Gil 2000). El depósito sedimentario de Lole-6 presenta un perfil expuesto en la barranca del zanjón que permitió, durante la prospección de 1993, que se observaran botones y restos faunísticos de clara asignación temporal posterior al contacto hispano indígena.

En 1994 se abrió una cuadrícula de 1,5 m x $2 \mathrm{~m}$ que lindaba contra la barranca. La excavación se profundizó hasta la roca estéril, unos $70 \mathrm{~cm}$ desde la superficie actual, extrayéndose niveles de $10 \mathrm{~cm}$ y $5 \mathrm{~cm}$. El sedimento fue zarandeado y se mapearon hallazgos 


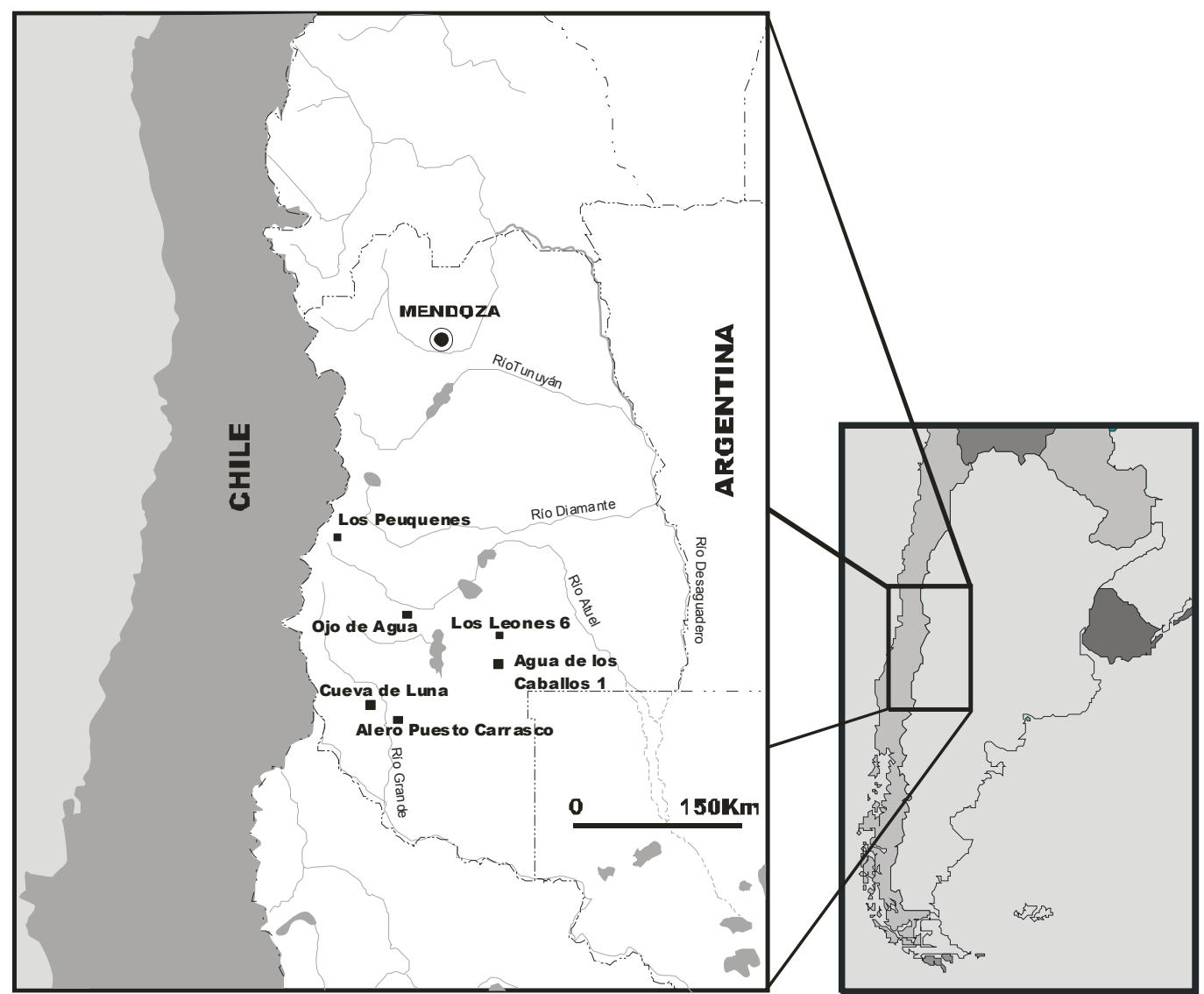

Figura 1. Localización de los sitios arqueológicos estudiados.

en planta. En la matriz sedimentaria no se observó una estratificación diferencial, y preliminarmente se descarta una importante acción fluvial en su conformación (Ermili com. pers. 1995). Los niveles superiores, primeros $20 \mathrm{~cm}$, sufrieron remoción en el sector contra el barranco, lo cual se nota en la pendiente de la superficie. A los fines de hacer comparable la información y dado que no se notaron diferencias estratigráficas relevantes se agruparon los datos en niveles de $10 \mathrm{~cm}$, excepto el último, nivel 7, que es de $5 \mathrm{~cm}$ de espesor. Considerando las características de la cultura material se asume que la ocupación humana en Lole-6 es asignable cronológicamente al período hispánico indígena, posiblemente del siglo XIX. La alta tasa depositacional permite explorar algunos cambios ergológicos con alta resolución temporal que en la región hasta ahora se desconocían para sitios a cielo abierto y para este período cronológico. Basados en observaciones estratigráficas y cambios en el tipo de hallazgos se postula que el registro es diacrónico, con buena resolución temporal, a lo largo del mencionado siglo. En los niveles superiores, primeras tres extracciones, se registran asociados loza blanca, vidrio y metal, elementos que están ausentes en los niveles inferiores, de la extracción 4 a la 7.

En Lole-6 se relevó información taxonómica, anatómica y tafonómica en 3.721 especimenes de los 6.863 recuperados. La Tabla 1 muestra la lista taxonómica con los valores de NISP. Se destaca que entre los taxones hay fauna doméstica euroasiática en todos los niveles del sitio. 


\begin{tabular}{|c|c|c|c|c|c|c|c|c|c|c|c|c|c|c|c|c|}
\hline & \multicolumn{7}{|c|}{ Los Leones-6 (Lole-6) } & \multicolumn{2}{|c|}{$\begin{array}{c}\text { Agua de Los } \\
\text { Caballos-1 } \\
\text { (ACA-1) }\end{array}$} & \multicolumn{2}{|c|}{$\begin{array}{l}\text { Ojo de Agua } \\
\text { (LB-1) }\end{array}$} & \multirow{2}{*}{\begin{tabular}{|c|}
$\begin{array}{c}\text { Los } \\
\text { Peuquenes } \\
\text { (LP) }\end{array}$ \\
- \\
\end{tabular}} & \multicolumn{2}{|c|}{$\begin{array}{c}\text { Alero Puesto } \\
\text { Carrasco (APC) }\end{array}$} & \multicolumn{2}{|c|}{$\begin{array}{l}\text { Cueva de } \\
\text { Luna (CL) }\end{array}$} \\
\hline & 1 & 2 & 3 & 4 & 5 & 6 & 7 & $1 / 4$ & $5 / 8$ & 1 & 2 & & $P C 1$ & $P C 2$ & CL1 & $C L 2$ \\
\hline No identificado & 92 & 210 & 1.334 & 717 & 447 & 423 & 128 & 215 & 764 & 174 & 1.038 & - & - & - & - & - \\
\hline Microvertebrado & & & & & & & & 52 & 144 & & & - & & & & \\
\hline Ave & - & - & - & - & - & - & - & - & 1 & - & 4 & 14 & 7 & 10 & - & - \\
\hline Ave gde. & - & - & 2 & 2 & 6 & 11 & 2 & - & - & - & 5 & - & - & - & - & - \\
\hline Emberizidae & - & - & - & - & - & - & - & - & - & - & - & - & - & - & - & 2 \\
\hline Zenaida auriculata & - & - & - & - & - & - & - & 1 & - & - & - & - & - & - & - & - \\
\hline Eudromia elegans & - & - & - & - & - & - & - & 1 & 4 & - & - & - & - & - & - & - \\
\hline Rehidae & - & 1 & - & - & - & - & - & 1 & 1 & - & 2 & - & 14 & 16 & - & 4 \\
\hline Rhea americana & - & - & - & - & - & - & - & - & - & - & 3 & - & 2 & 4 & - & - \\
\hline Pterocnemia pennata & - & - & - & - & - & - & - & - & 1 & - & - & - & 1 & 3 & 1 & - \\
\hline Mamífero & - & 30 & 135 & 31 & 29 & 39 & 14 & 2 & 5 & - & 17 & 72 & 207 & 332 & - & - \\
\hline Mamifero pequeño & - & - & - & - & - & - & - & 1 & 1 & - & - & - & - & - & - & - \\
\hline Mamifero mediano & - & - & - & - & - & - & - & 3 & 3 & - & - & - & - & - & - & - \\
\hline Mamifero grande & - & - & - & - & - & - & - & 16 & 15 & - & 24 & 91 & - & - & - & - \\
\hline Dasipodidae & 1 & $5(107)$ & $26(403)$ & $24(641)$ & $24(875)$ & $35(831)$ & 12(312) & $1(40)$ & $2(18)$ & $1(3)$ & $7(72)$ & - & 11 & 31 & 1 & 8 \\
\hline Euphratyni & - & - & - & - & - & - & - & - & - & - & - & - & 3 & 4 & 2 & 1 \\
\hline Chaetophractus $s p$. & - & - & - & - & - & - & - & - & - & - & - & - & 5 & 1 & 4 & 7 \\
\hline $\begin{array}{l}\text { Chaetophractus } \\
\text { villosus }\end{array}$ & - & - & - & - & - & - & - & - & - & - & - & - & 4 & 2 & 9 & 15 \\
\hline $\begin{array}{l}\text { Chaetophractus } \\
\text { villerosus }\end{array}$ & - & - & - & - & - & - & - & - & - & - & - & - & - & 3 & - & - \\
\hline Zaedyus pichyi & - & - & - & - & - & - & - & - & - & - & - & - & 21 & 34 & - & 2 \\
\hline Carnivora & - & - & 1 & - & - & - & - & - & - & - & - & - & 5 & 8 & - & - \\
\hline Felidae & - & - & 1 & - & - & - & - & - & - & - & - & - & - & - & - & - \\
\hline Felis concolor & - & - & - & - & - & - & - & - & - & - & - & - & - & - & - & 1 \\
\hline Canidae & - & - & - & - & - & - & - & - & - & - & - & - & - & 3 & 1 & - \\
\hline Conepatus $s p$. & - & - & - & - & - & - & - & - & - & - & - & - & - & 6 & 1 & - \\
\hline Artiodactylia & - & - & 1 & - & - & - & 1 & 1 & - & - & - & 15 & 59 & 62 & 2 & 12 \\
\hline $\begin{array}{l}\text { Ozotoceros } \\
\text { bezoarticus }\end{array}$ & - & - & - & - & - & - & - & - & - & - & - & - & - & 2 & - & - \\
\hline Camelidae & - & - & - & - & - & - & - & - & - & - & - & - & 37 & 42 & - & 1 \\
\hline Lama sp. & - & - & - & - & - & - & - & 11 & 12 & - & 1 & 72 & - & - & - & - \\
\hline Lama guanicoe & - & 1 & 7 & 3 & 2 & - & - & - & - & - & 3 & 66 & 329 & 370 & 58 & 82 \\
\hline Bos taurus & 7 & 13 & 14 & 3 & 5 & 2 & 3 & - & - & 1 & $\mid-$ & - & - & - & - & - \\
\hline Caprininae & 7 & 2 & 26 & 11 & 11 & 17 & 3 & - & - & 1 & 3 & - & 2 & 1 & 5 & 1 \\
\hline Equus cabalus & 1 & \begin{tabular}{|l|l}
1 \\
\end{tabular} & - & - & 1 & - & - & - & - & - & 16 & - & - & - & - & 4 \\
\hline Equus/Bos & - & - & 6 & 2 & 2 & - & - & - & - & - & 2 & - & - & - & - & - \\
\hline Herbivora & - & - & - & - & - & - & - & - & 3 & - & - & 2 & - & - & - & - \\
\hline Testudinidae & - & - & - & - & - & - & - & 1 & 3 & - & - & - & - & - & - & - \\
\hline TOTAL & 108 & 371 & 1.956 & 1.173 & 1.402 & 1.378 & 475 & 346 & \begin{tabular}{|l|}
977 \\
\end{tabular} & 180 & \begin{tabular}{|l|}
1.197 \\
\end{tabular} & 332 & 417 & 922 & 84 & 134 \\
\hline
\end{tabular}

Tabla 1. Detalle de los especímenes atribuidos a las distintas categorías taxonóicas de los conjuntos presentados. Entre parentesis se indican cantidades de placas dérmicas 


\section{Sitio Agua de Los Caballos-1 (ACA-1).}

Este sitio, es un abrigo rocoso que se emplaza en la margen izquierda del arroyo homónimo, en las proximidades del cerro Nevado (Gil 2000, 2002) (Figura 1). Luego de un sondeo realizado en los inicios de los años '80 se concretó la excavación durante el verano de 1996. Las primeras ocupaciones del abrigo se remontan a unos 1200 años AP. En términos generales se ha propuesto una distinción entre los materiales en cuatro grupos de niveles (Gil 2000). Teniendo en cuenta la cronología 14C y los objetivos del presente trabajo aquí se consideran los restos registrados en los niveles 1-4 y 5-8, comprendidos en los últimos 350 años 14C AP. La Tabla 1 detalla el análisis sobre los 1.323 especímenes, de los cuales 344 pudieron identificarse en algún nivel taxonómico. Si bien es notoria la alta cantidad de especímenes óseos no identificados también es clara la relativa diversidad taxonómica y la abundancia mayoritaria de camélidos. Se desprende de la tabla que las identificaciones realizadas no registran presencia de especímenes atribuidos a taxa domésticas euroasiáticas.

\section{Sitio Ojo de Agua (LB-1).}

Este sitio se localiza en el piedemonte del sur mendocino, a 35 $10^{\prime} 20^{\prime \prime} \mathrm{LS}$ y $69^{\circ} 39^{\prime} 45^{\prime \prime} \mathrm{LO}$ y cerca de Laguna Blanca y de El Sosneado (Figura 1). Durante los trabajos de prospección llevados a cabo en el área se observó la presencia de material óseo y lítico aflorando en el perfil del barranco que, según relatos de lugareños, se conformó en la última década por acción de frecuentes crecidas del arroyo "Ojo de Agua" (Neme 2002). En el sitio se excavaron dos cuadrículas, A1 y A2, ubicadas en ambas márgenes del cauce, abarcando en total unos $5 \mathrm{~m} 2$ de superficie. En el sitio, pero no en estas cuadrículas, se extrajeron esqueletos humanos con una cronología aproximada a los 1200 años AP (Novellino y Neme1999). La muestra extraída de la unidad de excavación A2, sobre la margen izquierda del arroyo, es la que se considera para este estudio pues en ellas se registró material atribuible a época hispano indígena.

En la unidad A2 se excavaron $4 \mathrm{~m} 2$ hasta una profundidad de $105 \mathrm{~cm}$. Si bien los restos asignables al período hispano indígena representan una capa de $30 \mathrm{~cm}$ de espesor, el conjunto de restos óseos no tiene más de $8 \mathrm{~cm}$. de espesor y que buza con dirección suroestenoreste. Así es que a medida que avanzaban los trabajos de excavación la presencia del estrato con alta frecuencia de restos óseos se desplazaba hacia los niveles inferiores de la pared nordeste. La concentración principal de restos arqueológicos, básicamente restos óseos, corresponde a los niveles 12, 13, 14, 15, 16 y 17 (Tabla 1).

El material óseo de la cuadrícula A2 ha sido dividido en dos conjuntos e incluye 1.377 especímenes de los cuales se identificaron 248 (Tabla 1). El primer conjunto, denominado Conjunto 1, abarca los 10 primeros niveles $(80 \mathrm{~cm})$ y en él aparece una menor cantidad de material probablemente depositado durante el siglo XX, ya que entre los elementos recuperados se encontró plástico y metal. El segundo, denominado Conjunto 2, corresponde a la concentración de materiales que aparecen en un espesor de alrededor de $30 \mathrm{~cm}$ y es por eso que para su agregación se siguió un criterio estratigráfico. Este conjunto se desarrolla desde los $80 \mathrm{~cm}$ hasta los $120 \mathrm{~cm}$ y temporalmente correspondería al siglo XIX.

\section{Sitio Los Peuquenes (LP).}

El sitio Los Peuquenes (LP) se emplaza en el sector cordillerano del sur mendocino, a unos $3000 \mathrm{msnm}$, en el valle del río Atuel (Figura 1). El sitio está conformado por nueve estructuras 
rocosas semicirculares distribuidas en una superficie estimada de $2.500 \mathrm{~m} 2$ y que contiene ocupaciones fechadas en los 360 años 14C AP correspondiendo, por algunos indicadores arqueológicos, a los siglos XVII y XVIII. Los trabajos de campo permitieron excavar dos de estas estructuras en las cuales se obtuvieron numerosas evidencias arqueológicas con cronologías posteriores al contacto hispano-indígena (Neme 2002). Este sitio tiene claras evidencias de relaciones con poblaciones trascordilleranas manifestadas con el hallazgo de tiestos de cerámicos probablemente producidos en la vertiente occidental de los Andes (Neme 2002). Se recuperaron alrededor de 20.000 especímenes óseos pero sólo 332 de ellos pudieron ser identificados en algún nivel. La Tabla 1 muestra el registro faunistico recuperado en los mencionados trabajos de campo y se destaca la ausencia de animales domésticos.

\section{Alero Puesto Carrasco (APC).}

Este alero que se emplaza en la margen izquierda del río Grande (Figura 1) ha sido excavado desde 1987 en diversas campañas (Durán 2000). Se ha estudiado la totalidad de la muestra ósea, proveniente de unos $5 \mathrm{~m} 2$ de superficie. Si bien con los materiales registrados se definieron 6 componentes culturales (Durán 2000; Durán et al. 1999; Neme et al. 1999) aquí se presentan aquellos asignados a ocupaciones posteriores al contacto hispano indígena y que estratigráficamente provienen de la unidad PC1 y PC2 (Neme et al. 1999). Según Durán (2000) corresponden a las capas superiores (IV y V), cuya cronología inicial sería de aproximadamente 500 años AP. Considerando todos los conjuntos se registraron 3.085 especímenes óseos. La Tabla 1 presenta los datos de las dos unidades recientes, donde se observa la presencia de fauna euroasiática aunque en escasa abundancia.

\section{Cueva de Luna (CL).}

Esta cueva se encuentra en la margen derecha del río Grande (Figura 1), a unos $27 \mathrm{~km}$ al sur de Bardas Blancas (Neme et al. 1996). Se han excavado 3,5 $\mathrm{m} 2$ de donde proviene el registro arqueológico que permitió dividir el depósito en cinco componentes culturales (Durán 2000; Neme et al. 1996). Las ocupaciones humanas se inician unos 3.800 años 14C AP hasta épocas posteriores al contacto hispano-indígena. Siguiendo el criterio cronológico adoptado para el presente trabajo aquí se detalla la información proveniente de los dos componentes más recientes cuya cronología relativa mínima estimada los atribuye al siglo XVIII-XIX (Neme et al. 1996). En el sitio se recuperaron 7.780 especímenes óseos (Neme et al. 1996). En la Tabla 1 se detalla la información faunística de esos dos componentes donde se registran animales domésticos euroasiáticos.

\section{INCORPORACIÓN DE FAUNA DOMÉSTICA EUROASIÁTICA.}

La Tabla 1 presenta la lista taxonómica y la abundancia de especímenes provenientes de los sitios antes presentados. En la mencionada tabla se nota una heterogénea distribución en la presencia de los animales domésticos euroasiáticos. La Figura 2 muestra la tendencia cronológica de los taxa domésticos euroasiáticos. El registro de Lole-6 en el área de El Nevado presenta fauna doméstica en todos sus niveles, desde el inicio de la ocupación, aproximadamente en el siglo XIX. En el depósito se identificaron restos de Bos taurus, Equus caballus y Caprininae, taxa domésticas incorporadas luego del contacto europeo. El sitio próximo, ACA-1 no registra este tipo de animales. En el piedemonte cordillerano, cerca de El Sosneado, el sitio Ojo de Agua muestra presencia de taxa doméstica en las dos unidades definidas (Figura 2). En la unidad más antigua, denominada unidad 2 y asignada tentativamente al 
siglo XIX, registra Caprininae, Equus caballus y Equus/Bos. La unidad más reciente, probablemente de los inicios del siglo XX, registra Bos taurus y Caprininae (Figura 2). En la cordillera, esta fauna no se registra. El valle del río Grande incluye dos sitios con ocupaciones registradas en el lapso temporal definido. Ambos tienen fauna doméstica pero mientras uno de ellos, APC, registra solo caprinos, el otro, CL, tiene también caballo.

La tendencia temporal señala una tardía incorporación de la vaca y el caballo en el registro arqueológico (siglo XIX), aunque una presencia de caprinos desde los inicio del contacto hispano-indígena. Hay dos sitios en cuestión, Lole-6 y LB-1, que permiten profundizar algunas cuestiones de la tendencia temporal de los animales domésticos euroasiáticos. Ambos sitios son del siglo XIX, y el segundo incluye una ocupación probablemente de inicios del siglo XX. Para Lole-6, la Figura 3 señala una mayor importancia relativa de la fauna silvestre, al inicio de la ocupación (en la Figura 3 a la derecha del conjunto), respecto a la doméstica. Este predominio se invierte a partir del nivel 3 donde la fauna domestica es, en términos de NISP, más abundante que la silvestre. El cambio definido en el nivel 3 es concordante con la incorporación de loza blanca, vidrio, metal y otros elementos en el registro arqueológico del Lole-6. En los niveles superiores la fauna silvestre está mínimamente presente respecto a la abundancia de especímenes de animales domésticos en esos niveles. El otro sitio, LB-1, señala una alta importancia de la fauna doméstica, mayor que la silvestre. Si se aceptan las cronologías asignadas para estas ocupaciones, entonces el registro señala que hacia finales del siglo XIX se nota una mayor importancia de la fauna doméstica sobre la silvestre (Figuras 2 y 3 ).

Esta variabilidad puede estar reflejando la funcionalidad de los sitios ya que algunos de ellos son depósitos a cielo abierto (LB-1, LOLE-6), otros son conjuntos que provienen de estructuras (LP), mientras que los restantes son registros de abrigos rocosos (ACA-1, CL, y $A P C)$. Se resalta que los dos sitios a cielo abierto son los que presentan la mayor diversidad de especies domésticas euroasiáticas, mientras que los restantes, en ningún caso, alcanzan a incluir el mismo número de especies. La Figura 3 muestra esta relación, basada en el NISP, entre animales domésticos y silvestres. Para elaborar esta figura se considera aquellos especímenes cuyas categorías taxonómicas pueden ser consideradas excluyentemente como doméstico o silvestre. Los sitios señalan una mayor importancia de animales domésticos en los sitios del piedemonte y las tierras bajas, mientras que en el valle del río Grande y la Cordillera la importancia parece haber sido menor, concordante con las expectativas presentadas por Prieto y Abraham (1993-1994).

\section{LA FAUNA DOMESTICA Y SU RELACION CON LA SILVESTRE}

Prieto y Abraham (1993-1994) plantean a la incorporación de ganado como uno de los factores intervinientes en el proceso de desertificación del sur mendocino. En ese marco toma relevancia analizar cómo se modificaron las estrategias con la incorporación de la fauna domestica, qué impacto pudo ocasionar sobre un ecosistema que careció de esta fauna hasta la llegada de las poblaciones hispanas. Con ese objetivo se compara la composición de fauna silvestre dentro de cada depósito (Figura 4).Las categorías de pequeño, mediano y grande se definen según el peso siguiendo las especificaciones de Neme (2002). En los conjuntos donde la fauna doméstica es significativa, y que a la vez son sitios a cielo abierto y del siglo XIX (Lole-6 y LB) registran un predominio de animales silvestres pequeños respecto a los grandes y medianos. En los otros sitios la fauna doméstica está ausente o minoritariamente representada, y en ellos la fauna silvestre es mayoritariamente de tamaño grande (ACA-1, LP, APC, y CL). 


\begin{tabular}{|c|c|c|c|c|}
\hline \multicolumn{5}{|l|}{ Caprininae } \\
\hline \multicolumn{5}{|l|}{ Bos tawus } \\
\hline \multicolumn{5}{|l|}{ Equis cabalus. } \\
\hline \multicolumn{5}{|l|}{ Bos/Equus } \\
\hline & Siglo XVI & Siglo XVII & Siglo XVIII & Siglo XIX \\
\hline
\end{tabular}

Figura 2. Tendencia temporal, sehalada como presencia, de fauna doméstica no americana segûn el registro arqueofaunistico.

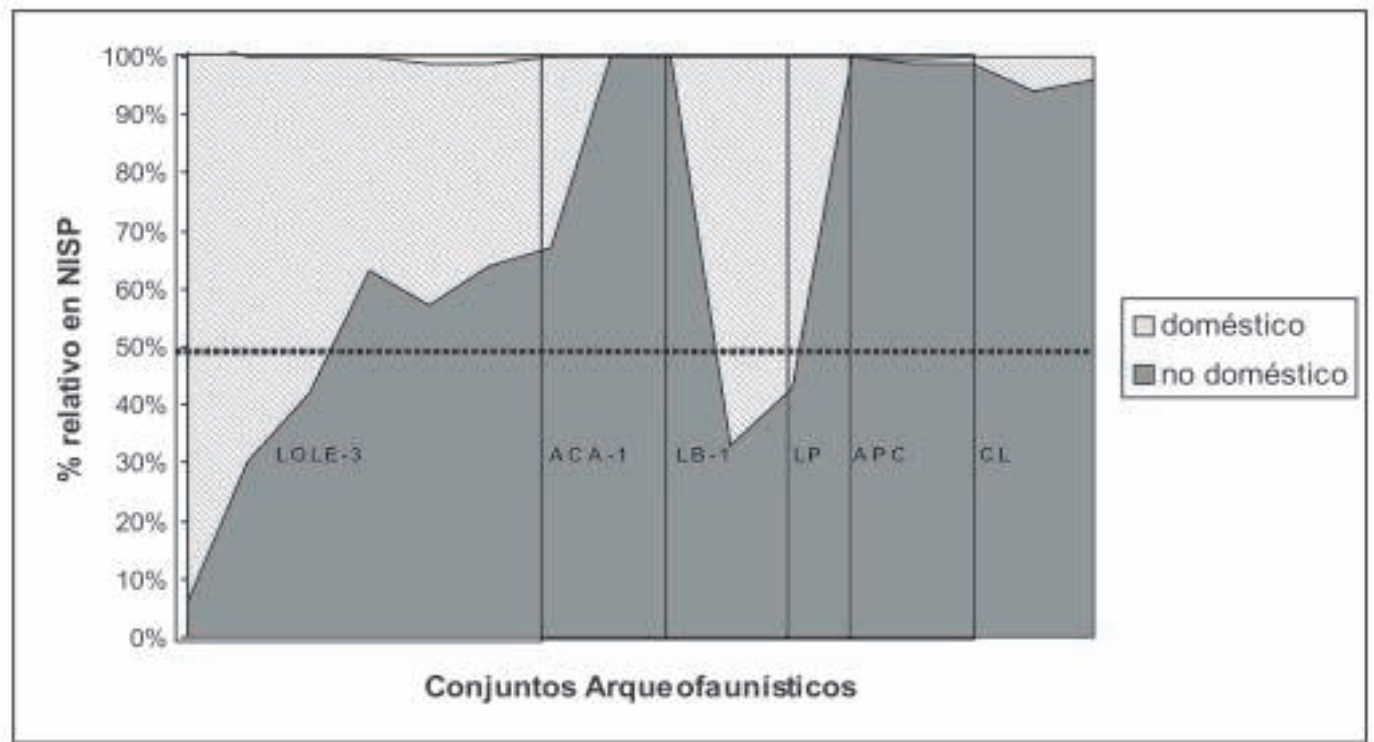

Figura 3. Proporción relativa doméstico/silvestre en los distintos conjuntos arqueofaunísticos analizados. En cada conjunto a la derecha las muestras más antiguas y a la izquierda las más recientes.

Profundizando el estudio de esta tendencia se realizó un análisis de regresión entre la proporción de animales domésticos vs. la proporción de silvestre pequeño (Figura 5). La primera variable es en relación a la muestra total mientras que la segunda es respecto al total de los silvestres. La figura muestra una significativa tendencia entre ambas variables donde el $74 \%$ de la variación porcentual de animales silvestres pequeños queda explicada por la variación en la proporción de fauna doméstica respecto a la fauna silvestre. Esto señala una tendencia a aumentar la proporción de silvestres pequeños cuando aumenta la proporción de animales domésticos.

En la Figura 5 se observan conjuntos que presentan más animales silvestres pequeños de los esperados, y otros donde se registran menos de los esperados. Entre los primeros sobresalen Lole-6 (2) y ACA-1 (1) mientras que entre los que registran menos fauna silvestre pequeña de la esperada se destaca LB-1(2). Esta relación directa entre la proporción de fauna doméstica y la proporción de animales silvestres pequeños señala un patrón significativo que puede interpretarse usando algunos principios de la ecología humana. Los dos primeros sitios (Lole-6 y ACA-1) se emplazan en el ecosistema del 


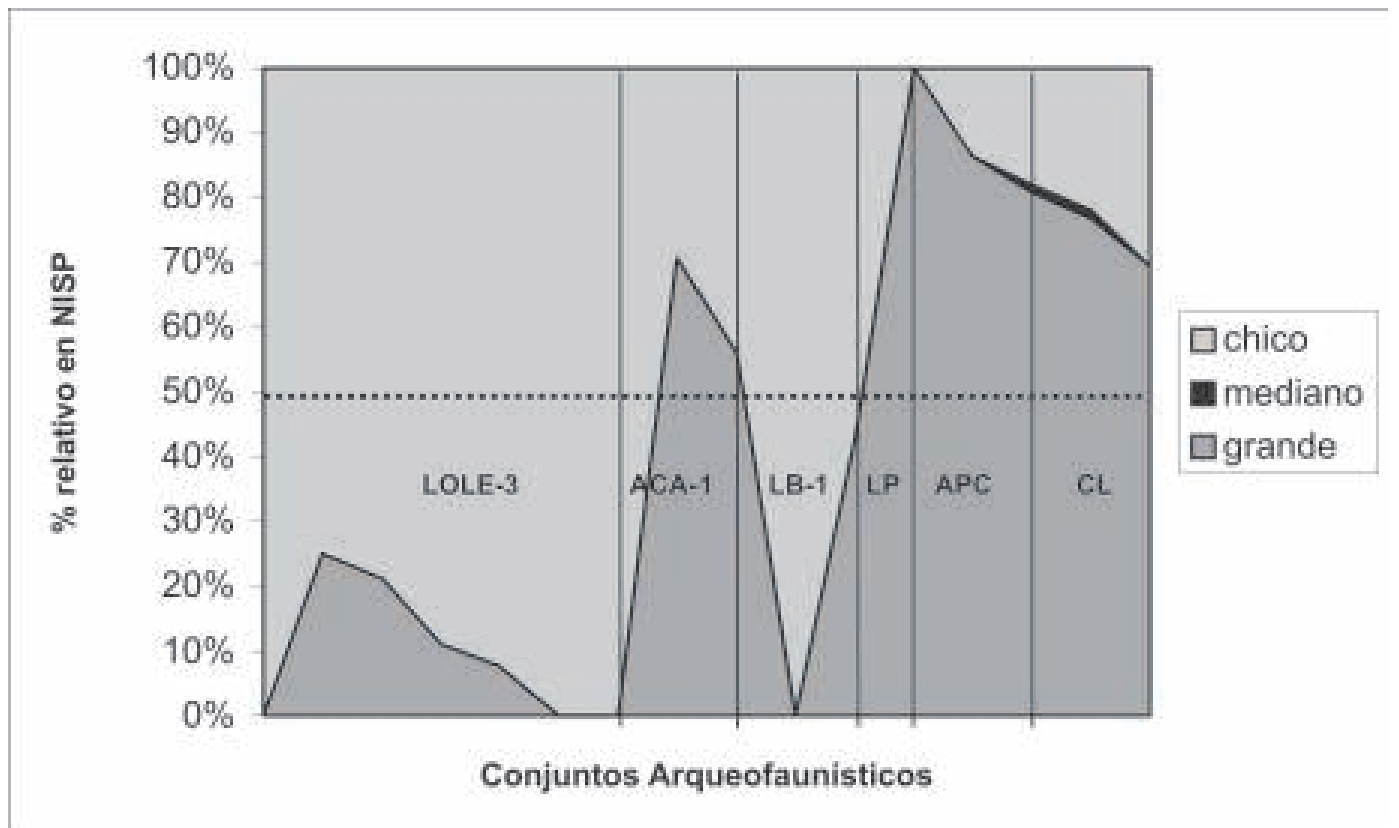

Figura 4. Proporción de distintas categorías de animales silvestre (grande, mediano y chico). En cada conjunto a la derecha las muestras más antiguas y a la izquierda las más recientes.

Monte y el segundo en el pedemonte próximo a la transición vegetacional Monte/ Patagonia. El modelo de amplitud de dieta presenta componentes fuertes para entender esta tendencia. Para este modelo los forrajeadores enfrentan elementos que pueden variar en abundancia, cantidad de energía producida por el elemento, cantidad de energía necesaria para adquirir el elemento y la cantidad de tiempo requerido para adquirirlo (Bettinger 1991). Entre las distintas implicancias del modelo, Bettinger (1991) resalta que la abundancia de un recurso no influye sobre su inclusión o no en la dieta óptima no que esa dedición depende de la abundancia de recurso de rango más alto respecto de su rendimiento energético. Otra implicancia que remarca es que a medida que la abundancia de un recurso decline aumenta el tiempo de búsqueda y en compensación aumenta la amplitud de dieta. Ya que no se cuentan con valores ajustados para discutir estas implicancias si se puede hipotetizar en base a ellas. Primero, los dos hábitats presentan una estructura de recursos diferencial en la que los animales domésticos podrían haber sido más rápidamente asimilados en aquellos hábitats donde la captura de fauna silvestre grande tendría un menor retorno energético, como hipotéticamente puede ser la situación del Monte respecto a otros hábitats como el pedemonte. Cabe destacar que la proporción de guanaco respecto a las otras especies silvestre incluso previo a la incorporación de ganado europeo es menor en el primer hábitat que en el segundo y la diversidad de especies sigue esa misma tendencia (Neme y Gil 2005). Esta tendencia podría facilitar la incorporación de fauna doméstica en el Monte que en los otros hábitats. Segundo la incorporación del ganado doméstico produjo una variación en los retornos de las presas vinculados con el impacto en el entorno que produce la incorporación de estos herbívoros que compiten con otros como el guanaco lo que cambiaría los valores de búsqueda. Para esta segunda alternativa Prieto y Abraham (1993-1994: 15) proponen el inicio, hacia fines del siglo XVII, de un proceso de competencia entre ganado asilvestrado y guanacos por las aguadas del sur este mendocino. A medida que aumenta la proporción 
de fauna doméstica ésta provoca la retracción de poblaciones silvestres competidoras aumentando en abundancia relativa los pequeños animales silvestres como los dasipódidos. El establecimiento de una explotación pastoril produce el distanciamiento de sus competidores, colocándolos en un orden inferior de preferencia dado su mayor costo de explotación (menos abundancia, mayores distancias para la búsqueda), situación que no sucedería con los pequeños animales silvestres que continuarían en el área con un bajo impacto por las incorporaciones faunísticas domésticas. En los contextos con poca o nula presencia de fauna doméstica, los animales grandes son mayoritarios respecto a la fauna silvestre pequeña.

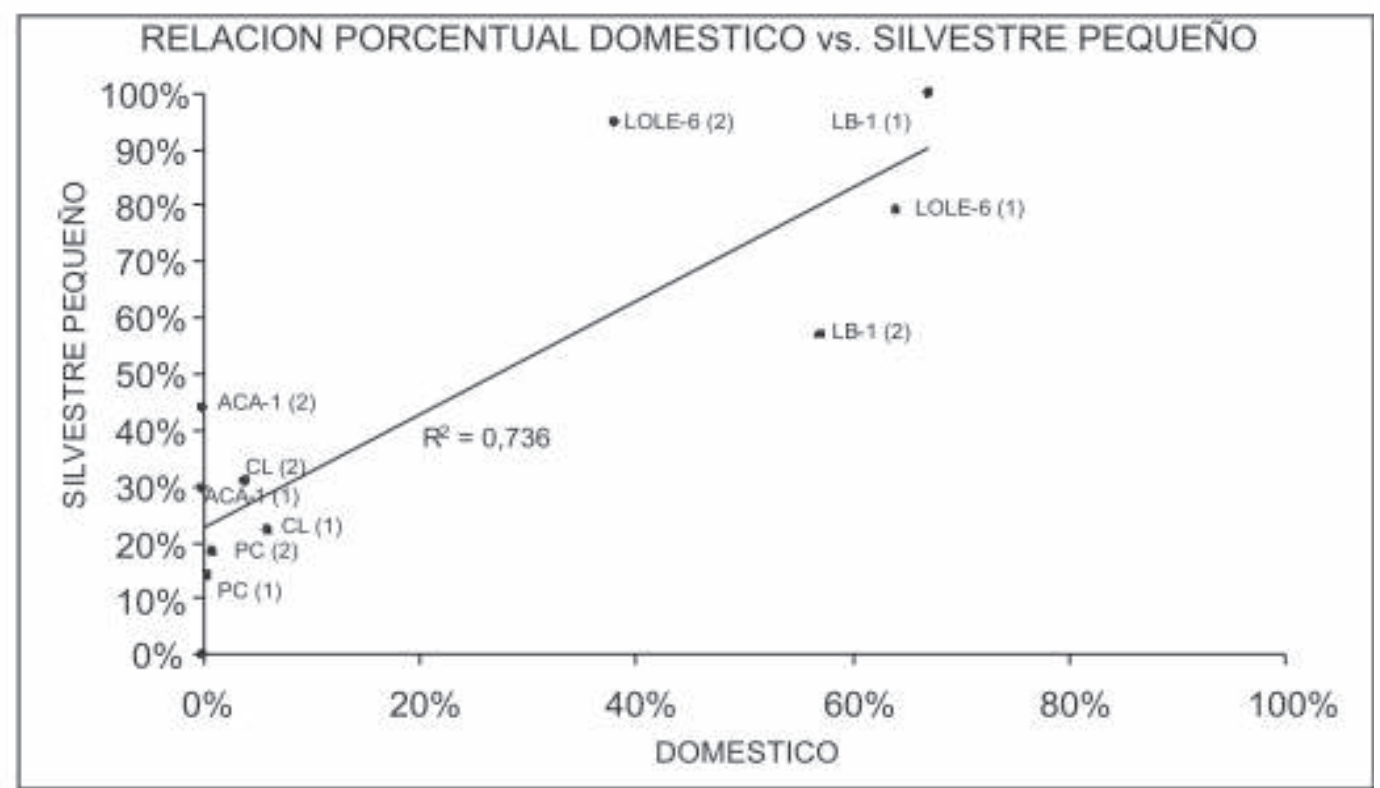

Figura 5. Análisis de regresión entre las proporciones respecto al total de fauna silvestre de fauna doméstica vs. fauna silvestre pequeña (ver explicación en el texto).

\section{CONCLUSION}

Prieto y Abraham (1993.1994) plantean como hipótesis que durante el siglo XVIII y comienzos del XIX se ejerció en el sur mendocino una fuerte presión antrópica sobre el ambiente que era vulnerable a raíz de reiterados ciclos de sequía y bajas temperaturas. Para Prieto y Abraham (1993-1994) esta doble presión de eventos climáticos y procesos antrópicos habrían determinado el inicio de la desertificación en el área. Sobre la base de la información escrita definen dos períodos. El primer período, desde aproximadamente 1650 hasta 1774 (siglo XVII y XVIII) está caracterizado por un uso extensivo del ecosistema con un avance relativo de la frontera agropecuaria hispana. Hacia el año 1658 se abandonan estos territorios por parte de las avanzadas españolas y se produce un asilvestramiento de ganado mayor esparciéndose por las planicies orientales. Este asilvestramiento y dispersión habría generado en esta planicie oriental un competencia por recursos y agua entre el ganado asilvestrado y los camélidos. El segundo período, comprendido entre 1774 y 1805 (fines del XVIII-inicios del XIX), fecha de instalación de fuertes, registra grandes cambios en los ecosistemas, manifestado por ejemplo en la ausencia 
de avistamiento de guanacos en el este. El registro arqueofaunístico señala que la fauna doméstica empezó a ser abundante en el siglo XIX, sobre todo en la planicie, y que hacia fines del siglo se habría convertido en el principal recurso animal.

El registro histórico señala que desde el siglo XVI las poblaciones locales del sur mendocino introdujeron ganado euroasiático, hay mención de caballo y perro, en sus sistemas culturales y con lo cual se habría iniciado un proceso de cambio también en el ambiente. Ambos probablemente fueron utilizados para las actividades de caza. Para estas fechas no hay citas concretas de cría de estos animales. El registro arqueofaunístico presenta muy débiles señales de estos procesos, y limitado hasta el momento a los caprinos. La información sobre cría de animales domésticos se encuentra en los documentos del siglo XVIII (Durán 1996), e incluyen ovinos, caprinos, vacas y equinos aunque este elenco se ve arqueológicamente registrado, si se aceptan las fechas asignadas a los sitios en consideración, hacia el siglo XIX. Esto refleja los distintos grados de resolución entre ambos registros y no necesariamente implican que uno de ellos esté "en lo cierto". Entre las diferencias se cuentan la menor resolución temporal del registro arqueológico respecto a la documentación escrita y el promedio en el largo plazo de las estrategias humanas reflejadas en el primer registro sobre la descripción particular en un punto del espacio y tiempo en el segundo tipo de registro.

La información presentada señala variabilidad en cuanto a las taxa domésticas registradas por distintos sitios y en cuanto a la proporción de éstas respecto a la fauna silvestre. El área de El Nevado, para el siglo XIX tiene un importante predominio de ganado doméstico, incluyendo los tres taxa: vaca, caballo y caprinos. En los otros sectores se registra una ausencia de esta fauna (cordillera, sitio Los Peuquenes) o la ausencia de vaca (valle del río Grande) mientras que aunque El Sonseado (sitio Ojo de Agua) también registra fauna doméstica ésta no es relativamente menos abundante que en Los Leones, registrando vaca sólo el nivel más reciente. En ese sentido el registro señalaría que la incorporación del ganado no fue un proceso homogéneo y simultáneo sino más bien un proceso heterogéneo.

Finalmente un punto a destacar es la correlación positiva entre la proporción de animales domésticos y silvestres pequeños. En ese sentido la información disponible señalaría que la incorporación del ganado euroasiático produjo un cambio en el ecosistema, al menos de competencia entre la fauna, que habría implicado o un cambio en los costos de caza de fauna silvestre o una disminución en la explotación de éstas en beneficio de los animales euroasiáticos.

\section{AGRADECIMIENTOS}

Este estudio se realizó en el marco de los proyectos subsidiados por la Fundación Antorchas (14116-116 y 12248-139) y la Agencia Nacional de Promoción Científica y Tecnológica (IM36 N0412750). Una versión del trabajo fue presentado en el $1^{\circ}$ Congreso Nacional de Arqueología Histórica (Mendoza 2000). Agradecemos a Mariana Mondini por sus sugerencias sobre el texto, a los evaluadores consultados por la revista y a quienes participaron en las tareas de campo, muchos para detallar aquí. 


\section{BIBLIOGRAFIA}

Bettinger, R.

1991 Hunter-Gatherer: Archaeological and Evolutionary Theory. Plenum Press.

Cabrera, Pablo

1929 Los Aborígenes del país de Cuyo. Revista de la Universidad Nacional de Córdoba. 15/ 16 (7-10; 1-8). Córdoba.

Durán, Víctor.

1994 Las poblaciones indígenas del sur mendocino durante los siglos XVI y XVII. Anales de Arqueología y Etnología 46/47. Mendoza.

1996 La araucanización de las poblaciones indígenas del sur mendocino (siglos XVIII y XIX). Anales de Arqueología y Etnología 48/49: 31-55. Mendoza.

1999 Los pehuenches malargüinos. Una aproximación histórica y su contrastación arqueológica. Revista de Estudios Regionales No19: 119-161. Mendoza.

2000 Poblaciones Indígenas de Malargüe. Su arqueología e historia. CEIDER Serie Libros No1. Mendoza.

Durán, Victor, Gustavo Neme, y Adolfo Gil

1999 Algunos problemas relacionados con el registro arqueológico de Alero Puesto carrasco (curso medio del valle del río Grande, Malargue, Mendoza). Soplando el Viento...Actas de las terceras Jornadas de Arqueología de la Patagonia, pp.:333-355. Bariloche.

Gil, Adolfo

2000 Arqueología de La Payunia. Tesis Doctoral. Fac. de Cs. N. y Museo. UN de la Plata. 2002 El registro arqueológico y la ocupación humana de La Payunia. En: Gil, A. y G. Neme (Eds.) Entre Montañas y Desiertos: Arqueología del sur de Mendoza, pp.:103-118. Sociedad Argentina de Antropología, Buenos Aires.

Lagiglia, Humberto

1983 Primeros contactos hispanicos-indígenas de Mendoza (La arqueología histórica y su periodificación). En: Morresi, E. y R. Gutierrez (Eds.) Presencia Hispánica en la Arqueología Argentina 1: 205-227. Museo Regional de Antropología "Juan A. Martinet"; Universidad Nacional del Nordeste. Resistencia; Chaco.

León Solís, Leonardo.

1991 Maloqueros y conchavadores en Araucanía y Las Pampas, 1700-1800. EdicionesUniversidad de La Frontera. Temuco, Chile.

Mandrini, Raúl.

1991 Procesos de especialización regional en la economía indígena pampeana (s. XVIIIXIX): el Caso del suroeste bonaerense. En: Boletín Americanista Nº41. Barcelona.

Michieli, Catalina

1978 Los Puelches. Publicaciones 4. Instituto de Investigaciones Arqueológicas y Museo. Universidad Nacional de San Juan. 
Neme, Gustavo

2002 Arqueología del Alto valle del río Atuel: Modelos, problemas y perspectivas en el estudio de las regiones de altura del sur de Mendoza". En: Gil, A. y G. Neme (Eds.) Entre Montañas y Desiertos: Arqueología del sur de Mendoza, pp.: 64-83. SAA. Buenos Aires.

Neme, G., V. Durán y A. Gil

1996 Análisis Arqueofaunístico del Sitio "Cueva de Luna" (Malargüe - Mendoza; Argentina). Hombres y Desierto. Actas XIII Congreso Nacional de Arqueología Chilena, pp.: 363370. Antofagasta. Chile

Neme, Gustavo, Adolfo Gil y Victor Durán

1999 El Registro Arqueofaunístico del Alero Puesto Carrasco (Malargüe-Mendoza). Solando el Viento...Actas de las Terceras Jornadas de Arqueología de la Patagonia, 491514. Bariloche.

Neme, Gustavo y Adolfo Gil

2002 La explotación faunística y la frecuencia de partes esqueletarias en el registro arqueológico del sur mendocino. En: Gil, A. y G. Neme (Eds.) Entre Montañas y Desiertos: Arqueología del sur de Mendoza, pp.: 141-155. Sociedad Argentina de Antropología, Buenos Aires. 2005 Faunal Exploitation and Agricultural Transition in the South American Agricultural limits. Trabajo presentado en el $70^{\circ}$ Meeting Society of American Archaeology. Utah.

Novellino, Paula y Gustavo Neme

1999 Hallazgos de restos esqueletales humanos en laguna Blanca y El Sosneado (sur de Mendoza). Acta del XII Congreso Nacional de Arqueología Argentina, 256-260. La Plata.

Prieto, María.

1984 Formación y consolidación de una sociedad en el Area Marginal del Reino de Chile. Tesis doctoral. España.

Prieto, María y Elena Abraham.

1993-1994 Indios, chilenos y estancieros en el sur de Mendoza. Historia de un proceso de desertificación. Anales de Arqueología y Etnología 48-49:11-29. Mendoza

Prieto, María, P. Dussel y G. Scodeller.

1999 Frontera y relaciones interétnicas en Mendoza: los pehuenches y el poder colonial entre 1780 y 1810. Informe Secretaría de Ciencia y Técnica. Universidad Nacional de Cuyo. Inédito. Mendoza.

Schiffer, Michael

1987 Formation Processes of Archaeological Record. University of new México Press.

Villalobos, Sergio.

1989 Los pehuenches en la vida fronteriza. Ediciones Universidad Católica de Chile, Santiago de Chile

Yellen, John

1990 The present and the future of hunter-gatherer studies. En: Lamberg-Karlovsky C. C.

(Ed.) Archaeological thought in America. Cambridge Press. 